\title{
Classical stochastic diffusion theory for thermal desorption from solid surfaces
}

\author{
Antonio Redondo \\ Electronics Division, Los Alamos National Laboratory, Los Alamos, New Mexico 87545 \\ Yehuda Zeiria) and William A. Goddard III \\ Arthur A. Noyes Laboratory of Chemical Physics, California Institute of Technology, Pasadena, California \\ 91125 \\ (Received 31 January 1984; accepted 24 April 1984)

\begin{abstract}
As a first step in the microscopic study of dynamic processes on surfaces and at interfaces, we have considered the thermal desorption of adsorbed species on solid surfaces. We review recent developments based on a classical stochastic diffusion formulation. Using this theory, we obtained a simple rate expression, $R=\left(\Omega_{0} / 2 \pi\right) f(T) \exp \left(-D_{e} / k T\right)$, where $\Omega_{0}$ is the surfaceadsorbate vibrational frequency and $D_{e}$ the dissociation energy. For atoms $f(T)=1$, whereas for molecules $f(T)$ depends on the parameters for the frustrated rotations at the surface. The effect of coverage on the rate of desorption and the process of desorption into a fluid are also examined. Finally, we discuss the relationship between our theory and the expressions obtained from
\end{abstract} \\ activated complex (transition-state) theory.
}

PACS numbers: 68.45.Da, 82.65.My

\section{INTRODUCTION}

The understanding of dynamical processes occurring on the surfaces and at interfaces of solids is crucial for the development of theoretical and experimental techniques in a wide range of technological applications, such as crystal growth, heterogeneous catalysis, and electrochemistry. As a first step in the study of such dynamical phenomena, we have considered one of the simplest and most basic processes: the thermal desorption of adsorbed species from solid surfaces. Due to the development of accurate experimental techniques [e.g., molecular beam relaxation spectrometry (MBRS), ${ }^{\mid(|a|}$ temperature programmed desorption (TPD), ${ }^{\text {(it) }}$ low energy electron diffraction (LEED), ${ }^{2(c)}$ Auger electron spectroscopy (AES), ${ }^{1 /(d)}$ thermal desorption mass spectrometry (TDS), ${ }^{(1)}$ etc.], a vast literature related to adsorption-desorption experiments is available for a large variety of systems. The need for a basic understanding of the nature of such processes and the availability of experimental data have stimulated the proposal of many theoretical methods and models. ${ }^{2-6}$

The most commonly used framework for the discussion of desorption is a statistical thermodynamical approach based on transition state theory (TST). ${ }^{2}$ Although this method correctly accounts for the observed magnitude of atomic desorption rates, quantitative predictions have been difficult since it requires knowledge of the transition state for the desorbing species. These problems have been particularly serious for desorption of molecules.

Although TST had not provided a prediction of specific desorption rates, it did provide a justification for the Arrhennius form

$$
R=A \exp \left(-E_{d} / k T\right)
$$

usually used in characterizing experimental desorption rates in the limit of low coverages (no interaction between the adparticles).
A number of authors ${ }^{3}$ have combined transition state theory with stochastic trajectory calculations for the evaluation of desorption rates. The theories that employ stochastic trajectory calculations are computationally involved and hence difficult to apply directly. There are also microscopic theories $^{4}$ in which an explicit coupling between the adparticle and the surface phonon modes is introduced to calculate the energy flow between the surface and the adparticle. However, these microscopic approaches lead to discrepancies between experimental and calculated desorption rates for physisorbed atoms. Moreover, there appears to be difficulties in applying these approaches to molecular desorption.

The experimental and theoretical studies undertaken over the last two decades have led to a better understanding of the desorption mechanisms. They have, however, simultaneously raised some additional basic questions. One puzzling result pertains to the measurements ${ }^{1(\mathbf{a}) / 5}$ of both $A$ and $E_{d}$ for the desorption of $\mathrm{CO}$ from different metal surfaces. Although the observed values for the preexponential factor (in the limit of low coverage) vary over three orders of magnitude, all of the experimental results indicate that the values of $A$ are at least two orders of magnitude larger than the corresponding values observed for atomic systems $\left(A \simeq 10^{13}\right.$ $\left.\mathrm{s}^{-1}\right)$. In addition, several experiments ${ }^{5(\mathrm{~b})}$, $S_{(\mathrm{c})}$ lead to different functional relationships between $A$ and $E_{d}$ and the surface coverage.

These and other examples have illustrated the need for theoretical methods that yield explicit expressions for the rate of desorption in terms of the microscopic properties (e.g., vibrational frequencies, bond energy, etc.) and dynamics of the adsorbate-surface system.

In the present paper, we review a theoretical description for desorption" which leads to a simple rate expression given in terms of the microscopic properties of the system. The rate of desorption is found to have the form 


$$
R=\frac{\Omega_{0}}{2 \pi} f(T) r(T) \exp \left(-\frac{D_{e}}{k T}\right),
$$

where $T$ is the temperature, $k$ is Boltzmann's constant, $D_{e}$ is the bond enthalpy, and $\Omega_{0}$ is the surface adsorbate vibrational frequency. For atoms, the factor $f(T)=1$, but for molecules $f(T)$ depends on the parameters for the frustrated rotations at the surface. For molecules this factor can be $10^{2}$ to $10^{3}$, leading to the much larger desorption rates observed experimentally. The function $r(T) \simeq 1$ for desorption into vacuum, but it depends on the microscopic parameters of the system when the particle is desorbing into a liquid lvide infra). This method is based on a classical stochastic diffusion theory (CSDT) which follows a procedure proposed by Kramers ${ }^{8}$ but modified so as to be appropriate for desorption. We also examine the effect of coverage ${ }^{7(c)}$ on the rate of desorption, as well as the problem of desorption into a fluid phase. ${ }^{9}$ Finally, we consider the relationship between the present treatment and transition state theory. ${ }^{10}$

\section{DERIVATION OF THE EXPRESSION FOR THE RATE OF DESORPTION}

\section{A. Stochastic equation of motion?}

We start by considering the relative motion of the adparticle with respect to the surface atoms. In so doing, we follow the ideas introduced by Adelman and Doll. ${ }^{11,12}$ We consider explicitly the motion of a few surface atoms that are strongly interacting with the adsorbed particle, while the rest of the crystal is assumed to act as a heat bath. Without loss of generality, we shall consider the motion of only one surface atom

We first assume that the adparticle is an atom; a generalization of the results for the case of molecules will be considered below. A further simplification is introduced by assuming a one-dimensional system in which both the adatom and the surface atom are restricted to move in a direction normal to the surface. Thus, the motion of the adatom will be described by

$$
m \ddot{z}=-\frac{\partial V(z-\zeta)}{\partial z},
$$

where $z$ and $\zeta$ are the positions of the adatom and the surface atom, respectively, $m$ is the mass of the adatom and $V(z-\zeta)$ is the interaction potential between the adatom and the surface.

The motion of the surface atom will be described by a generalized Langevin equation, ${ }^{11}$

$$
\begin{aligned}
m_{s} \ddot{\xi}= & -\frac{\partial V(z-\xi)}{\partial \xi}-m_{s} \omega_{s}^{2} \xi \\
& -m_{s} \int_{0}^{t} \theta\left(t-t^{\prime}\right) \xi\left(t^{\prime}\right) d t^{\prime}+\tilde{f}(t),
\end{aligned}
$$

where $m_{\mathrm{s}}$ represents the mass of the surface atom; $\omega_{\mathrm{s}}$ is the characteristic frequency of the solid, $\theta\left(t-t^{\prime}\right)$ and $f(t)$ correspond to a memory kernel and random force, which include the influence of the heat bath on the motion of the surface atom. The functions $\theta(t)$ and $\tilde{f}(t)$ are related by the second fluctuation-dissipation theorem. ${ }^{12}$

Integrating the memory kernel in Eq. (II.2) by parts and taking the Markovian limit ${ }^{11,13}$ one obtains a Langevin equation of motion for the surface atom,

J. Vac. Scl. Technol. B, Vol. 2, No. 3, July-Sept. 1984

$$
m_{s} \ddot{\xi}=-\frac{\partial V(z-\zeta)}{\partial \zeta}-m_{s} \Omega_{s}^{2} \xi-m_{s} \beta_{s} \dot{\xi}+f(t),
$$

where $\Omega_{s}$ is the effective frequency, $\beta_{s}$ is the friction constant, and $f(t)$ is a random force with a Gaussian distribution. ${ }^{8,11}$ The interaction potential $V(z-\xi)$ between the adatom and the surface is often described in terms of Morse or Lennard-Jones-type potentials, leading to nonlinear equations of motion.

The problem is then linearized by means of a parabolic splines fit $\left(A+B z+1 / 2 C z^{2}\right.$ in each interval) to the potential $V(z-\zeta)$. Thus, the formal solution of Eq. (II.3) by means of Laplace transforms " is now possible, leading to

$$
\begin{aligned}
m \ddot{z}(t)= & -\frac{\partial V}{\partial z}\left(1-\frac{C}{m_{s} \Omega^{2}}\right) \\
& -\frac{C^{2}}{m_{s}} \int_{0}^{t} \Lambda\left(t^{\prime}\right) \dot{z}\left(t-t^{\prime}\right) d t^{\prime}+C R(t),
\end{aligned}
$$

where $\partial V(z) / \partial z=B+C z$ and

$$
\begin{aligned}
\Lambda(t)= & \frac{1}{Q} \int_{t}^{\infty}\left\{\exp \left[-\left(\beta_{s}-Q\right) \frac{t^{\prime}}{2}\right]\right. \\
& \left.-\exp \left[-\left(\beta_{s}+Q\right) \frac{t^{\prime}}{2}\right]\right\} d t^{\prime},
\end{aligned}
$$

with $Q^{2}=\beta_{s}^{2}-4 \Omega_{s}^{2}-4 C / m_{s} ; R(t)$ includes the initial conditions and the random force $f(t)$. [See Ref. $7(\mathrm{~b})$ for a derivation of Eq. (11.4)].

We now take the Markovian limit of the convolution integral in Eq. (II.4). This approximation is valid in the present case because the time $t$ appearing in the integral refers to characteristic desorption times. On the other hand, from Eq. (II.5), we see that the decay time for $\Lambda(t)$ is $\beta_{s}^{-1}$. To estimate $\beta_{s}$, we use the Debye approximation, which gives ${ }^{11}$

$$
\beta_{s}=(\pi / 6) \omega_{D} \text {, }
$$

where $\omega_{D}$ is the Debye frequency of the surface. The time $\beta_{s}^{-1}$ is then of the order of magnitude of the period of a molecular vibration so that $t>\beta_{s}^{-1}$. We can then put $z\left(t-t^{\prime}\right) \simeq z(t)$ and let $t \rightarrow \infty$ in the upper limit of the convolution integral, so that Eq. (II.4) becomes

$$
m \ddot{z}(t)=-\frac{\partial V}{\partial z}\left(1-\frac{C}{m_{s} \Omega^{2}}\right)-\frac{C^{2} \beta_{s}}{m_{s} \Omega^{4}} \ddot{z}(t)+C R(t),
$$

where $\Omega^{2}=\Omega_{s}^{2}+C / m_{s}$.

The first term in the right-hand side of Eq. (1I.7) is the product of the force at point $z,-\partial V / \partial z$, multiplied by a correction factor $\left(1-C / m_{s} \Omega^{2}\right)$. The force $-\partial V / \partial z$ is what would be obtained from theoretical calculations in which the surface atoms are kept fixed. The term $\left(1-C / m_{s} \Omega^{2}\right)$ represents a correction to the surface-adparticle force (arising through the coupling of the adparticle with the crystal) due to the motion of surface atoms. Thus, the directly calculated surface-adsorbate frequency would be $\omega_{0}=\left(C / m_{s}\right)^{1 / 2}$, however, the modification due to the motion of the crystal atoms leads to

$$
\bar{\omega}^{2}=\omega_{0}^{2}\left(1-\frac{C}{m_{s} \Omega^{2}}\right)=\omega_{0}^{2}\left(\frac{\Omega_{s}^{2}}{\Omega_{s}^{2}+\omega_{0}^{2}}\right)
$$

(a reduced frequency). This may explain why the calculated surface-adsorbate frequencies (i.e., $\omega_{0}$ ) are often larger than 
the experimentally observed values (i.e., $\omega$ ). ${ }^{1}$ In the limit $T \rightarrow 0$ the correction term becomes unity. ${ }^{7(b)}$

Equation (II.7) is a Langevin-type equation describing the motion of the adatom coupled to the solid surface, which acts as a heat bath. We can rewrite it in the form

$$
\ddot{z}=-\widetilde{B}-\widetilde{\Omega}^{2} z-\beta \dot{z}+\frac{C R(t)}{m} \text {. }
$$

\section{B. Rate of desorption for atoms ${ }^{7}$}

In a desorption process we are interested in time scales which are much larger than those characteristic of molecular motion $\beta^{-1}$ [see Eq. (II.8)]. Thus, in obtaining an expression for the desorption rate, we follow the derivation originally suggested by Kramers. ${ }^{8}$ We start by considering the generalized Liouville equation

$$
\frac{\partial W}{\partial t}+u \frac{\partial W}{\partial z}+K \frac{\partial W}{\partial u}=\beta u \frac{\partial W}{\partial u}+\beta W+q \frac{\partial^{2} W}{\partial u^{2}},
$$

where $W(z, u, t)$ is the probability distribution to find the particle at position $z$ with velocity $u$ at time $t$, and $q=k T \beta / m$. Here, $\beta$ is the friction constant in Eq. (II.8) and $K$ represents the acceleration caused by the interaction potential $\widetilde{V}$ coupling the adatom to the surface. This acceleration $K$ is given, according to Eq. (II.8), by

$$
K=-\left(\widetilde{\beta}+\tilde{\Omega}^{2} z\right)=-\frac{1}{m} \frac{\partial \widetilde{V}(z)}{\partial z} .
$$

Following the development of Chandrasekhar ${ }^{8(b)}$ one can show $^{7(b)}$ that the (steady state) expression for the flux of desorbing particles at a distance $Z_{0}$ from the surface is given by

$$
J\left(Z_{0}\right)=\int_{u_{0}}^{\infty} W\left(Z_{0}, u\right) u d u=C_{0} \frac{k T}{m} r(T) \exp \left(-\frac{D_{e}}{k T}\right),
$$

where

$$
D_{e}=\tilde{\boldsymbol{V}}\left(Z_{0}\right)+\frac{1}{2} m u_{0}^{2},
$$

and

$$
r(T)=\Phi\left(\alpha_{1}\right)+\exp (-\eta)\left(\frac{\pi \beta k T}{2 m a}\right)^{1 / 2}\left[1-\Phi\left(\alpha_{2}\right)\right],
$$

with

$$
\begin{aligned}
& \alpha_{1}=\left[\frac{m(a-\beta)}{2 k T \beta}\right]^{1 / 2}\left[u_{0}-\left(a Z_{0}+b\right)\right], \\
& \alpha_{2}=\left(\frac{m a}{2 k T \beta}\right)^{1 / 2}\left[-\theta\left(a Z_{0}+b\right)+u_{0}\right], \\
& \theta=1-\beta / a, \eta=\frac{\theta \beta\left(a Z_{0}+b\right)^{2}}{2 q} .
\end{aligned}
$$

The symbol $\Phi(\alpha)$ stands for the error function, $C_{0}$ is a normalization constant, and

$$
\begin{aligned}
& a=\frac{1}{2}\left[\beta+\left(\beta^{2}-4 \widetilde{\Omega}^{2}\right)^{1 / 2}\right] \\
& b=-\frac{2 \widetilde{B}}{-\beta+\left(\beta^{2}-4 \widetilde{\Omega}^{2}\right)^{1 / 2}} .
\end{aligned}
$$

The rate for a desorption process is given ${ }^{8}$ by the ratio of the flux at $Z_{0}$ to the number of particles $N_{0}$ at the surface. For experimentally interesting temperature ranges (where $\left.k T<D_{e}\right)$ one can approximate the interaction potential $\widetilde{V}(z)$ by a harmonic potential to obtain

$$
N_{0}=C_{0}\left(\frac{2 \pi k T}{m \Omega_{0}}\right)
$$

where $\Omega_{0}$ is the value of $\Omega$ [c.f. Eq. (II.8)] at the minimum. The final expression for the rate of desorption ${ }^{7(b)}$ is

$$
R=\frac{\Omega_{0}}{2 \pi} r(T) \exp \left(-\frac{D_{e}}{k T}\right) \text {. }
$$

\section{Rate of desorption for molecules?}

Next, we consider the case of a molecule desorbing from a solid surface. For such a system, in addition to the translational degree of freedom, there also exist frustrated motions, such as frustrated rotations and translations. For example, considering the diatomic molecule $\mathrm{CO}$, the most important frustrated motion corresponds to the bending mode of the oxygen atom about the surface-carbon bond. For the free molecule there is no direct coupling between the pure translational and the pure rotational motions, but for the adsorbed system the two motions are coupled through the surface. Thus, the total interaction potential between the molecule and the solid surface can be written in the form

$$
V_{\text {total }}=\tilde{V}(z)+V_{\text {rot }}(\gamma)
$$

where $\widetilde{V}(z)$ and $V_{\text {rot }}(\gamma)$ represent the potentials due to pure translation and frustrated rotation (i.e., the bending mode), respectively. In such a case, the molecules, which are in equilibrium with the surface, will have a Maxwell-Boltzmann distribution of frustrated rotational energy. The total distribution function, obtained as a solution to the generalized Liouville equation, will have the form

$W(z, u, \gamma, \dot{\gamma})=W(z, u) \exp \left(-\frac{\mu l^{2} \Omega_{r}^{2} \gamma^{2}+\mu l^{2} \dot{\gamma}^{2}}{2 k T}\right)$,

where $W(z, u)$ is the steady state solution of Eq. (II.9) and the harmonic approximation is used for the frustrated rotational motion ${ }^{14}$ (here $\mu l^{2} \Omega_{r}^{2}$ is the force constant of the frustrated rotation).

Due to the coupling of the translational and rotational motions to the surface, we assume that the energy stored in the frustrated rotation can be converted to translational kinetic energy of the desorbing molecule. As a result, the desorption energy $D_{c}$ is given by

$$
D_{e}=\widetilde{V}\left(Z_{0}\right)+\frac{1}{2} m u_{0}^{2}+\frac{1}{2} \mu l^{2} \dot{\gamma}^{2}+\frac{1}{2} \mu l^{2} \Omega_{r}^{2} \gamma^{2} .
$$

Following a procedure similar to that of the previous subsection, ${ }^{7(b)}$ we obtain [in the harmonic approximation for $V(z)$; for the general expression see Ref. 7(b)]

$$
R=\frac{\Omega_{0}}{2 \pi}\left(\frac{2 \mu l^{2} \Omega_{r}^{2} \gamma_{0}^{2}}{\pi k T}\right) r(T) \exp \left(-\frac{D_{c}}{k T}\right),
$$

where $\gamma_{0}$ is the maximum bending angle for the molecule. The choice of the value of $\gamma_{0}$ is somewhat arbitrary; however, it must be such that

$$
\exp \left(-\frac{1}{2} \mu l^{2} \Omega^{2}{ }_{r}^{2}\right)<1
$$


In the case of desorption into vacuum, the function $r(T)$ is practically unity (for the systems we have studied) so that the resulting expressions for the rate of desorption are ${ }^{7(b)}$

$$
\begin{aligned}
& R_{\text {atom }}=\frac{\Omega_{0}}{2 \pi} \exp \left(-\frac{D_{e}}{k T}\right), \\
& R_{\text {molecule }}=\frac{\Omega_{0}}{2 \pi}\left(\frac{2 \mu l^{2} \Omega_{r}^{2} \gamma_{0}^{2}}{\pi k T}\right) \exp \left(-\frac{D_{e}}{k T}\right) .
\end{aligned}
$$

\section{COVERAGE DEPENDENCE ${ }^{7(\mathrm{c})}$}

Adsorbate-adsorbate interactions influence the desorption rate via a modification of the interaction potential $V(z)$ (and hence $D_{e}$ ). For simplicity, in this section we assume that the direct adsorbate-surface interaction is not changed by the adsorbate-adsorbate interaction, so that the net potential seen by the desorbing particle is the superposition of its interaction with the surface and with the other adparticles. Although this assumption may not be valid in all cases (e.g., when three-body or charge transfer effects are important) it is likely to be fairly accurate for noble gas adsorbates. However, this assumption is not essential because the present method can be implemented to include more general potentials.

In order to incorporate coverage effects into $D_{e}$ and $V(z)$ we must determine the optimum (minimum free energy) arrangement of the adparticles on the surface. To do this, we first assume that the particles adsorb on well defined (periodic) sites on the infinite surface. Next, we choose a relatively small region of the surface, referred to as the desorption region, which is used to generate all possible arrangements of the adparticles for each coverage $\theta$. In order to determine the configuration(s) exhibiting the minimum free energy, one must calculate the total energy associated with each arrangement. Since the adparticle-surface interactions are the same for all arrangements (for a given $\theta$ ) their sum over all the adparticles in the system can be omitted from the calculation of the total energy, hence the total energy consists of a pairwise sum of adsorbate-adsorbate interaction energies. We divide this pairwise sum into two terms,

$$
E_{\text {total }}=\sum_{\text {desorption region }}\left(V_{\text {in-in }}+V_{\text {in-out }}\right) \text {, }
$$

where $E_{\text {toral }}$ is the total adsorbate-adsorbate interaction energy of the desorption region, $V_{\text {in-in }}$ represents the adsorbate interactions within the desorption region, and $V_{\text {in-out }}$ corresponds to the interaction energy of an adparticle inside the desorption region and all the other adparticles outside this zone. To evaluate the second term in Eq. (III.1) we use a mean field approximation in which each site outside the desorption region is assumed to contain $\theta$ adparticles (where $\theta$ is the coverage). The value of $E_{\text {total }}$, for the different configurations, is then calculated by combining an appropriate adsorbate-adsorbate interaction potential with the arrangement of the adparticles in the desorption region.

Once the total energies for all the possible arrangements (at a given $\theta$ ) have been evaluated, the configurations are subdivided into groups according to their $E_{\text {total }}$ values. The free energy associated with each group of configurations is then obtained, as a function of temperature, using

$$
F=E_{\text {total }}-T S
$$

and

$$
S=k \log (\Gamma),
$$

where $\Gamma$ is the number of configurations with energy $E_{\text {total }}$. The arrangement of adparticles (inside the desorption region) used to evaluate the desorption rate is chosen from the group of configurations corresponding to the lowest free energy. ${ }^{7(c)}$

After identifying the minimum free energy configuration(s) (for a given coverage and temperature range) we calculate the net potential felt by the desorbing particle as

$$
V_{\text {net }}(z)=\widetilde{V}(z)+\sum_{j=0}^{\text {adparticles }} V_{0 j}(z),
$$

where $\widetilde{\boldsymbol{V}}$ is the effective adsorbate-surface interaction potential for $\theta=0$ and $V_{0 j}$ is the pairwise interaction between the desorbing particle and the $j$ th adparticle on the surface. Finally, to obtain the temperature dependence of the rate of desorption for a given coverage, the potential energy in Eq. (II.8) is replaced by $V_{\text {net }}(z)$ and the $D_{e}$ by the corresponding well depth of the net potential. ${ }^{7(\mathrm{c})}$

From the coverage dependence of the desorption rate $R(\theta)$ we can extract the coverage dependence of the preexponential factor $A(\theta)$ and the effective dissociation energy $D_{e}(\theta)$. From these relationships we can calculate the temperature programmed desorption (TPD) spectra by solving the Redhead equation ${ }^{15}$

$$
\frac{d \theta}{d t}=-\theta A(\theta) \exp \left[-\frac{D_{e}(\theta)}{k T}\right]
$$

\section{DESORPTION OF ATOMS INTO LIQUIDS?}

The derivation of an expression for the rate of desorption into a liquid phase follows the same basic steps presented in Sec. II. In this case, however, one must also consider the interaction between the desorbing particle and the liquid molecules that surround it.

We start by considering a system composed of a desorbing particle (atom or ion) with mass $M$, interacting with a solid surface and a number of solvent particles, with mass $M_{l}$, as illustrated schematically in Fig. 1. We divide the substrate atoms into two groups: (i) a small number of atoms which are strongly interacting with the adsorbed particle, this group is called the primary zone, and (ii) the rest of the solid which is assumed to act as a heat bath. As in Sec. II, without loss of generality, we assume that the primary zone contains only

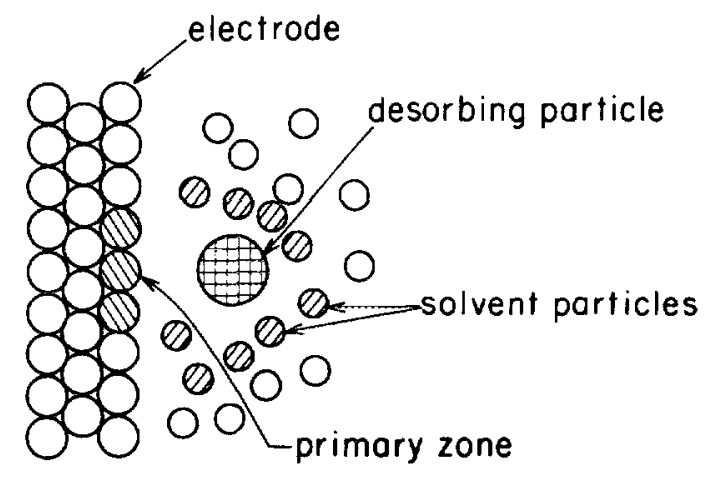

FIG. 1. Schematic description of the absorbate at a solid/liquid interface (after Ref. 9). 
one atom whose mass is denoted by $M_{s}$. We also simplify the problem by limiting both the desorbing particle and the primary zone atom to a one-dimensional motion normal to the solid surface. Thus, the motion of the adparticle will be described by a Newtonian equation of motion

$$
M \ddot{R}=-\frac{\partial V(R, \zeta, \mathbf{Z})}{\partial R},
$$

where $\zeta$ represents the displacement of the surface primary zone atom from its lattice point. The vector $\mathbf{Z}$ includes the displacement from equilibrium along the line connecting the centers of the adparticle and the solvent particles surrounding it. The total interaction potential $V(R, \zeta, \mathrm{Z})$ felt by the adparticle is assumed to be given by a sum of pairwise interactions

$$
V(R, \zeta, \mathbf{Z})=V(R-\zeta)+\sum_{i=1}^{N(R)} V_{i}\left(R-Z_{i}\right),
$$

where $N(R)$ is the number of solvent particles around the adsorbate when it is at a distance $R$ from the surface. Because the desorbing particle is always surrounded by a "cage" of liquid molecules, we use harmonic potentials to represent the interaction between the adparticle and the liquid,

$$
V_{i}\left(R-Z_{i}\right)=\frac{1}{2} k_{i}\left(R-Z_{i}\right)^{2} .
$$

The interaction between the surface and the desorbing particle is represented by a properly dissociating potential (e.g., Morse or Lennard-Jones). Again we fit this potential by parabolic splines.

The motion of the primary zone atom and that of the solvent molecules are described using the equivalent harmonic chain representation (EHCR) introduced by Adelman. ${ }^{16}$ This representation is used to construct model heat baths which, upon the application of the Markovian approximation, lead to Langevin-type equations. ${ }^{9}$ As before, the introduction of the Markovian approximation is justified by the large differences between the time scales for desorption and vibrations.

Thus, the equation of motion for the primary zone atom reduces to

$$
M_{s} \ddot{\zeta}=-M_{s} \Omega_{s}^{2 \zeta}-M_{s} \beta_{s} \dot{z}-\frac{\partial V(R, \zeta, \mathbf{Z})}{\partial \zeta}+f_{s}(t) .(\text { IV.3 })
$$

Similarly, the motion of the $i$ th solvent particle is described by

$$
M_{i} \ddot{Z}_{i}=-M_{i} \Omega_{i}^{2} Z_{i}-M_{i} \beta_{i} \dot{Z}_{i}-\frac{\partial V(R, \zeta, Z)}{\partial Z_{i}}+f_{i}(t) .
$$

The effective frequencies and friction constants of Eqs. (IV.3) and (IV.4) can be expressed in terms of the parameters of the EHCR, as shown in Ref. 9.

Next, we substitute the potentials of Eq. (IV.2) into Eqs. (IV.3) and (IV.4) and solve them formally (using Laplace transforms). Assuming that the adsorbate-liquid interaction has the same form for all the $N(R)$ solvent particles, we can rewrite the effective equation of motion for the desorbing particle as

$$
\begin{aligned}
M \ddot{R}= & -\left\{\left[N(R) k_{l}-\Gamma_{l}(0)\right]\right. \\
& \left.+\left[C(R)-\Gamma_{s}(0)\right]\right\} R-B(R) \\
& -\int_{0}^{t}\left[\Gamma_{l}\left(t-t^{\prime}\right)+\Gamma_{s}\left(t-t^{\prime}\right)\right] \dot{R}\left(t^{\prime}\right) d t^{\prime} \\
& -\left[\Gamma_{l}(t)+\Gamma_{s}(t)\right] R(0)+F(t),
\end{aligned}
$$

where

$$
\begin{aligned}
& \Gamma_{l}(t)=N(R) \frac{k_{l}^{2}}{M_{l}} \int_{t}^{\infty} \Lambda_{l}\left(t^{\prime}\right) d t^{\prime}, \\
& \Gamma_{s}(t)=\frac{C_{0} C(R)}{M_{s}} \int_{t}^{\infty} \Lambda_{s}\left(t^{\prime}\right) d t^{\prime},
\end{aligned}
$$

and where $\Lambda_{t}(t)$ and $\Lambda_{s}(t)$ have exactly the same form as the integrand of Eq. (II.5), with $Q_{l}^{2}=\beta_{l}^{2}-4 \widetilde{\Omega}_{l}^{2}, \widetilde{\Omega}_{l}^{2}=\Omega_{l}^{2}$ $+K_{l} / M_{l}, Q_{s}^{2}=\beta_{s}^{2}-4 \widetilde{\Omega}_{s}^{2}$, and $\widetilde{\Omega}_{s}^{2}=\Omega_{s}^{2}+C_{0} / M_{s}$. Here $C_{0}$ and $C(R)$ represent the force constants associated with the adparticle-surface and adparticle-liquid interactions; $B(R)$ is the corresponding linear coefficient of the parabolic spline fits to $V(R-\zeta)$, and $F(t)$ is a random force.

The term in braces in Eq. (IV.5) corresponds to the total potential felt by the desorbing particle. It includes the corrections due to the thermal motion of both the surface atoms and the solvent particles surrounding the desorbing particle; thus, this is the potential that one would obtain experimentally. We denote this total potential by $V_{\text {tot }}(R, \zeta, Z)$. Due to the large difference in time scales, one may introduce the Markovian limit to Eq. (IV.S) and obtain the corresponding Langevin equation

$$
M \ddot{R}=-\frac{\partial V_{\mathrm{tot}}(R, \zeta, Z)}{\partial R}-\left[\widetilde{\beta}_{i}(R)+\widetilde{\beta}_{s}(R)\right] \dot{R}+\widetilde{F}(t),
$$

where

$$
\begin{aligned}
& \tilde{\beta}_{l}(R)=\frac{N(R) k_{l}^{2} \beta_{l}}{M_{l} \Omega_{l}^{4}} ; \\
& \widetilde{\beta}_{s}(R)=\frac{C_{0} C(R) \beta_{s}}{M_{s} \widetilde{\Omega}_{s}^{4}} .
\end{aligned}
$$

Equation (IV.7) represents an effective equation of motion for the desorbing particle, which is coupled to a heat bath constituted by the solid surface and the liquid. Equation (IV.7) can thus be rewritten as

$$
M \ddot{R}=-\bar{B}(R)-\bar{C}(R) R-\beta_{\mathrm{tot}}(R) \dot{R}+\widetilde{F}(t),
$$

where $B(R)$ and $C(R)$ are the first and second derivatives of the total potential $V_{\text {tor }}$ evaluated at the point $R$, and

$$
\beta_{101}(R)=\beta_{l}(R)+\beta_{s}(R) \text {. }
$$

It describes the motion of the desorbing particle in terms of a Brownian oscillator in which the interaction with the heat bath is given in terms of the microscopic quantities of the system. The amplitude of the random force $\widetilde{F}(t)$ is defined by its relations to $\beta_{\text {tot }}$ through the second fluctuation-dissipation theorem.

Since Eq. (IV.8) has exactly the same form as Eq. (II.8), one can calculate the rate of desorption into the liquid using the procedure outlined in Sec. II B. Thus, we obtain

$$
R=\frac{\Omega_{0}}{2 \pi} r(T) \exp \left(-\frac{D_{e}}{k T}\right),
$$

where $r(T)$ is given by Eq. (II.12). As we shall see in the next section, contrary to the case of desorption into vacuum, this function is not necessarily equal to one. 


\section{RESULTS AND DISCUSSION}

\section{A. Desorption of atoms and molecules}

We shall now consider some examples of the application of the theory presented in the previous sections. In the case of atomic desorption we compare theoretical and experimental results for both chemisorption and physisorption (the two limits of strong and weak interaction potentials). For molecular desorption, we examine the desorption of $\mathrm{CO}$ from nickel surfaces.

In Fig. 2(a) we compare the calculated [using Eq. (II.17a)] and experimental ${ }^{17}$ rates of desorption as a function of temperature for chemisorbed $K$ on $W(111)$. In addition, the results calculated by De et al. ${ }^{4(\text { a) }}$ are shown. In these calculations we used the surface-adsorbate potential $V(z)$, obtained from the parameters of Ref. 4(a). For this system our results deviate from experiment by $\sim 0.2$ orders of magnitude, well within the experimental uncertainty.

The desorption rates of physisorbed $\mathrm{Xe}$ from $\mathrm{W}(111)$ are plotted in Fig. 2(b) with a comparison both to the experimen-
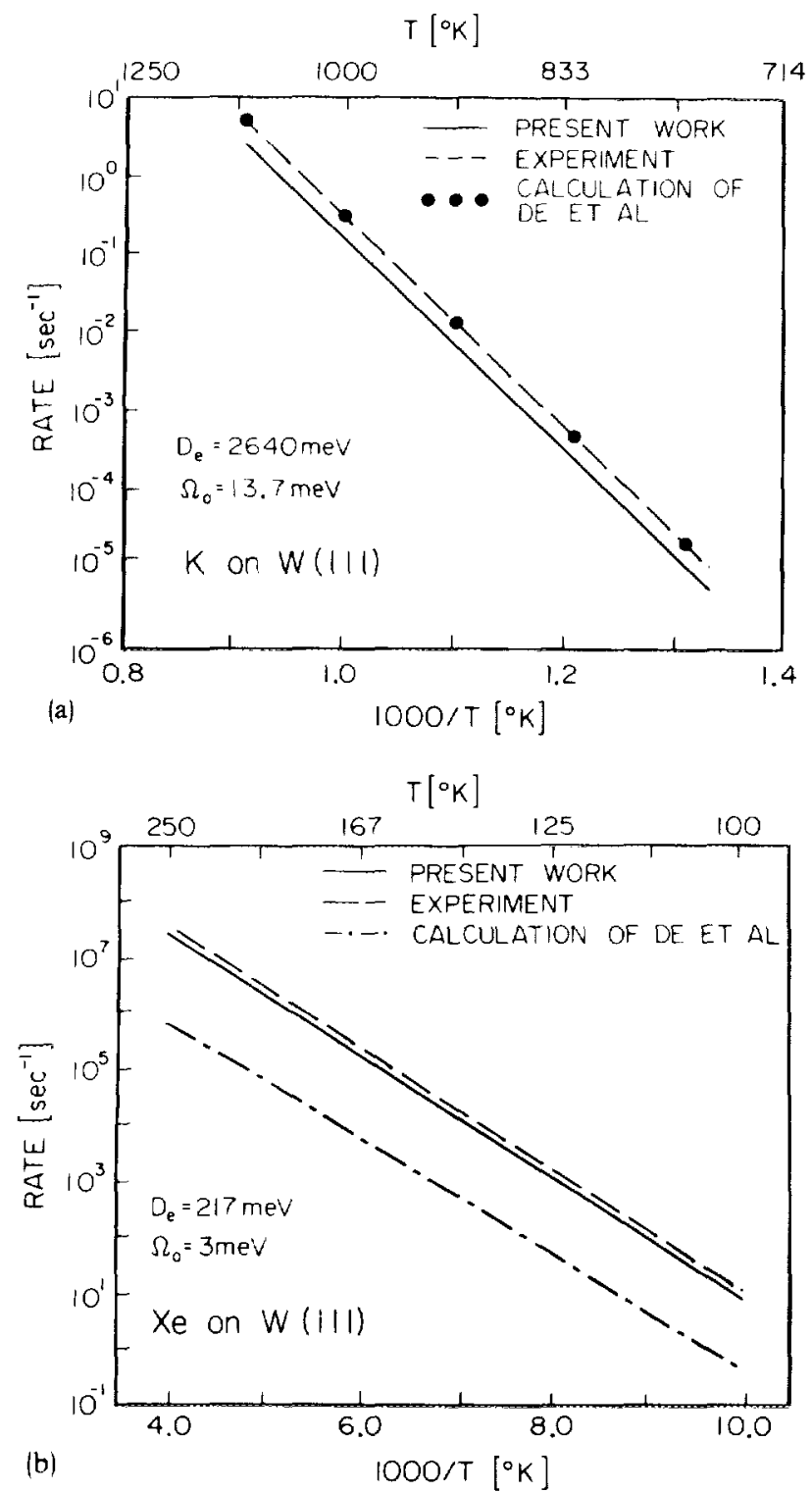

FIG. 2. (a) Desorption rates for a chemisorbed atomic system $K$ on $W(111)$; (b) desorption rates for a physisorbed atomic system Xe on W(111) [after Ref. 7(b)]. tal data ${ }^{17(c)}$ and to the calculations of De et al ${ }^{4(a)}$ Again we find that the desorption rate obtained from EQ. (1I.17a) is an excellent agreement with experiment.

These results clearly show that Eq. (II.17a) provides a very accurate description of the rate of desorption of atoms from solid surfaces. The simple relation between the rate of desorption $R$ and the characteristic microscopic parameters of the system $\Omega_{0}$ and $D_{e}$ makes it possible to predict one of the quantities from experimental determinations of the other two.

As an example of molecular desorption we have considered $\mathrm{CO}$ chemisorbed on $\mathrm{Ni}(110){ }^{7,18}$ In Fig. 3 we present the results of our calculations [Eq. (11.7b)], as well as the experimental values of Helms and Madix. ${ }^{1(a)}$ We also show the rate of desorption that one would obtain if the $\mathrm{CO}$ were treated as an atom [Eq. (II.17a)]. The excellent agreement between the theoretical calculation and the experimental data shown in Fig. 3 suggests that the frustrated rotational motion is responsible for the two order of magnitude increase in the preexponential factor.

\section{B. Coverage dependence}

To study the effect of coverage on the desorption rate within the framework described in Sec. III a model system was chosen. A difficulty associated with this type of study stems from the lack of quantitative experimental and theoretical data on the modifications to the adsorbate-surface and adsorbate-adsorbate interaction potentials due to changes in coverage. Such changes should become evident in systems where three-body interactions and/or charge transfer effects are important. In the present study we are mainly concerned with the qualitative behavior of the desorption rates as a function of coverage.

We have studied, as a model system, the desorption of $\mathrm{Xe}$ atoms from W(111) surfaces. Because of the weak bonding $\left(D_{e}=5 \mathrm{kcal} / \mathrm{mol}\right)$ we expect only a small overlap between the adsorbate and surface electronic wave functions, and hence the three-body and charge transfer effects should be

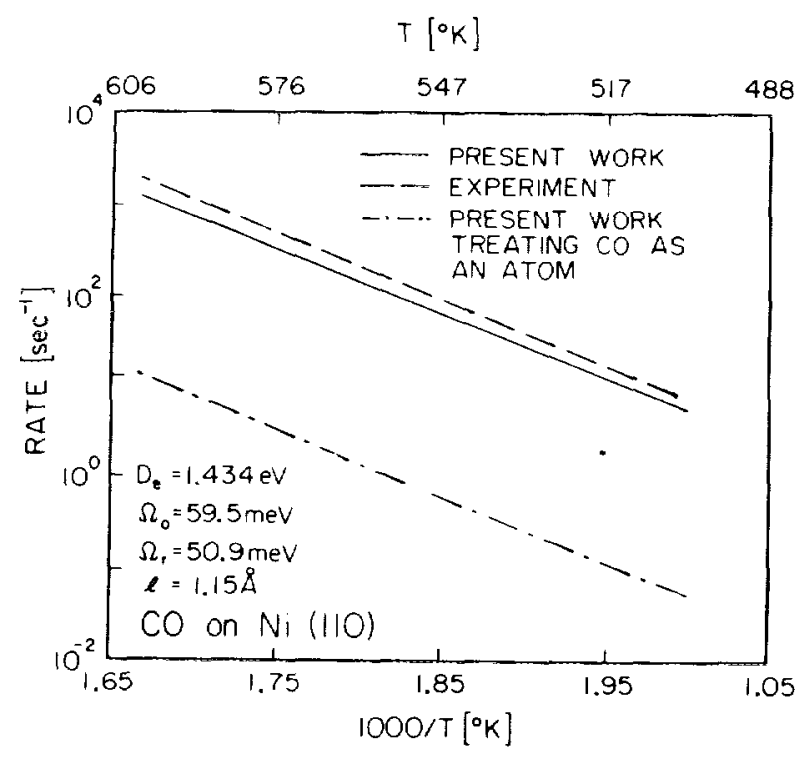

FiG. 3. Desorption rates for $\mathrm{CO}$ on $\mathrm{Ni}(110)$ [after Ref. 7(b)]. The experimental results are those of Helms and Madix [Ref. 1(a)]. 
negligible. Consequently, the adsorbate-surface interaction can be obtained from low coverage experimental results, ${ }^{19}$ however, the nature of the adsorbate-adsorbate interaction is not well known. We consider two limiting cases: (i) an attractive $\mathrm{Xe}-\mathrm{Xe}$ interaction obtained from gas phase scattering experiments ${ }^{20}$ and (ii) a purely repulsive $\mathrm{Xe}-\mathrm{Xe}$ interaction based also on experimental gas phase studies. ${ }^{21}$ The presence of the surface will probably make the effective XeXe interaction less attractive than in the gas phase so that the actual potential should be in between the above two cases.

In calculating the desorption rate, we always focus on a site that happens to be occupied, and we calculate the rate of desorption from this site. The specific arrangement of occupied sites used in calculating the TPD spectra is the most probable one (the lowest free energy) for a given coverage $\theta$ and temperature $T$. The details of this calculation are presented in Ref. 7(c).

For the attractive Xe-Xe interaction, a common characteristic is that for all the coverages studied the optimum arrangement (configuration) is independent of temperature over the range considered ( 50 to $150 \mathrm{~K}$ ). Essentially, at these temperatures, the system has condensed to the lowest enth alpy configuration. At higher temperatures the entropy would lead to a more dispersed arrangement of the adsorbed particles.

The results of the calculations show ${ }^{7(\mid)}$ that at a given temperature, the desorption rate increases with decreasing coverage. This behavior is a consequence of the attractive Xe-Xe interactions that lead to an increase of the effective bond energy as the coverage is increased. This trend is apparent from an examination of Fig. 4, where the variation in bond energy is given as a function of coverage. The dependence of the preexponential factor on the coverage is also shown in Fig. 4, where we find that $A(\theta)$ is essentially independent of $\theta$. Thus, one can assume a constant preexponential factor throughout the range of coverages studied. This behavior is somewhat surprising, since it suggests that the shape of the net potential [Eq. (III.3)] is independent of coverage. Hence, the most important factor governing the cover-

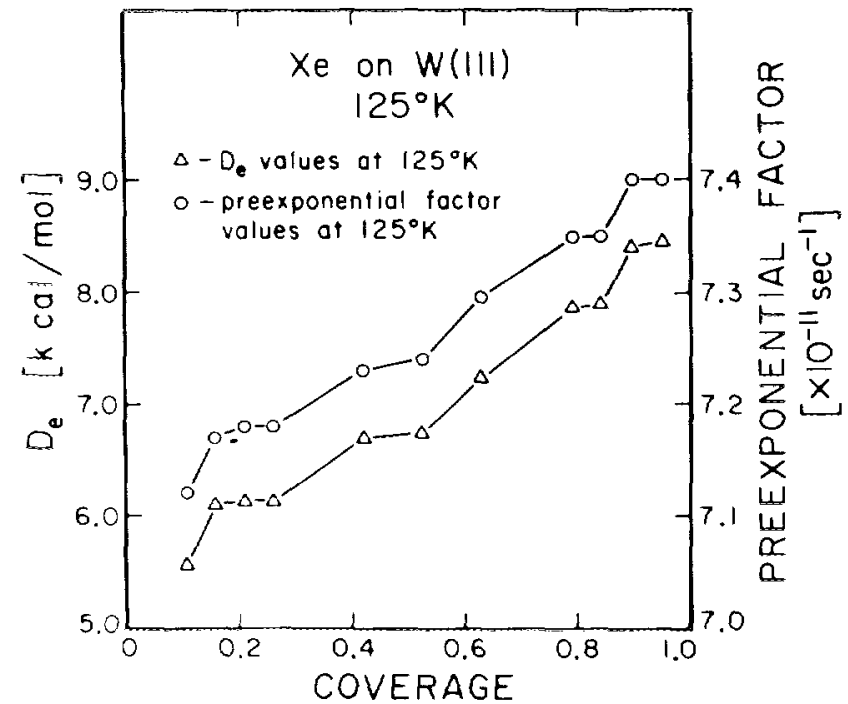

Fic. 4. Variation of the effective dissociation energy and the preexponential factor as a function of coverage for the desorption of Xe from W(11)/using the attractive $\mathrm{Xe}-\mathrm{Xe}$ interaction) at $125 \mathrm{~K}$ [after Ref. 7(c)] age dependence of the overall rate of desorption (for an attractive $\mathrm{Xe}-\mathrm{Xe}$ interaction) is the variation of the well depth.

In contrast with the results obtained for the attractive potential case, the repulsive $\mathrm{Xe}-\mathrm{Xe}$ interaction exhibits optimum (lowest free energy) configurations that depend on the temperature. In particular, for some coverages, it is possible to find two different optimal arrangements of adparticles in the desorption region, depending on the temperature. This phenomenon is due to the increasing importance of the entropy term in the expression for the free energy, Eq. (III.2).

In this case we find that, for a given temperature, the desorption rate increases with increasing coverage. This behav ior is opposite to that found for the attractive potential. As one would expect, the difference between the attractive and repulsive potentials is most marked for high coverages (e.g., for $\theta=0.95$ the rates of desorption differ by at least six orders of magnitude, whereas for $\theta=0.1$, they differ by $\sim 0.5$ orders of magnitude). Figure 5 shows the variation of the effective dissociation energies and preexponential factors as a function of coverage. Again we find that the dissociation energy has a strong dependence on the coverage, while the preexponential factor is practically constant. As a result, the dominant factor in the behavior of the total rate of desorption is the decrease of the effective dissociation energy with increas ing coverage.

\section{TPD spectra}

As discussed above, our calculations show (Figs. 4 and 5) that the preexponential factors for both the attractive and

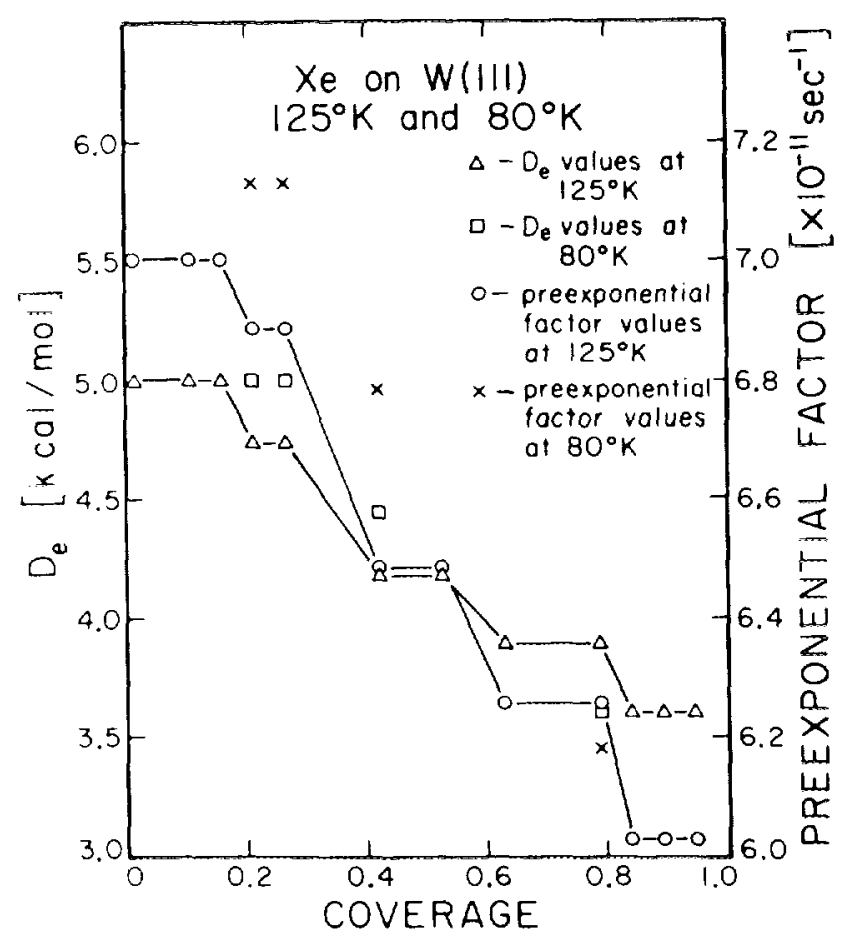

FIG. 5. Variation of the effective dissociation energy and the preexponentia factor as a function of coverage for the desorption of $\mathrm{Xe}-\mathrm{Xe}$ interaction at 125 and $80 \mathrm{~K}$. The preexponential factors and dissociation energies corresponding to the optimum configuration at $125 \mathrm{~K}$ correspond to the circles and triangles, respectively, and are connected by solid lines. The corre sponding quantities at $80 \mathrm{~K}$ are denoted by crosses and squares, respectively, (these are only shown when they differ from the respective values at 125 K) [after Ref. 7(c)]. 
repulsive $\mathrm{Xe}-\mathrm{Xe}$ interactions are practically coverage independent. Thus, we have taken $A(\theta)$ in Eq. (III.4) to be constant and equal to $7.0 \times 10^{11} \mathrm{~s}^{-1}$ for the attractive potential and $6.5 \times 10^{11} \mathrm{~s}^{-1}$ for the repulsive potential. Figures 4 and 5 show that the variation of $D_{e}$ with the coverage can be approximated by a linear relationship of the form

$$
D_{e}(\theta)=D_{c}^{0}+\beta \theta \text {, }
$$

where $D_{e}^{\circ}=0.217 \mathrm{eV}$ and $\beta_{\text {attractive }}=0.152 \mathrm{eV} ; \beta_{\text {repulsive }}$ $=-0.061 \mathrm{eV}$.

The TPD spectra corresponding to the attractive and repulsive potentials (with values of $\beta$ obtained from the results presented in Figs. 4 and 5) are shown in Figs. 6(a) and 6(b). Figure 6(c) shows the corresponding TPD curves for an attractive interaction $(\beta=0.02 \mathrm{eV})$ intermediate between those of Figs. $6(a)$ and $6(b)$. These results indicate that one can use this method to fit experimental data by varying the values of $A, D^{0}$, and $\beta$.

\section{Desporption into liquids}

One of the major difficulties in most microscopic theories of solid/liquid interfaces arises from the lack of reliable interation potentials for such systems. As an example, we have chosen a model potential of the Morse type.'

In Fig. 7 we present the calculated desorption rate as a function of temperature for two different values of $\Omega_{0}$ (all other parameters are kept constant). In both cases the solid line represents the actual rate while the dashed line represents the rate that one would obtain by setting $r(T)=1$. It is clear from these results that $r(T)$ is very sensitive to changes in the stretch frequency while its dependence on the variation in $T$ is weak. Moreover, from the functional form of $\gamma$ [Eq. (II.12)] one can see that this quantity will tend to reduce the desorption rate into a liquid with respect to that into vacuum. Figure 8 shows the variation of $\log (\Upsilon)$ as a function of the mass of the adparticle for different frequencies and bond strength of the total potential. This figure shows that $r(T)$ exhibits a strong dependence on $M, \Omega_{0}$, and $D_{e}$, while it is weakly dependent on both $D_{l}$ (in the physically meaningful range of the diffusion constant, $\left.5 \times 10^{-6}-5 \times 10^{-5}\right)$ and $T$. Because $r(T)$ differs greatly from unity and depends sensitively upon various parameters, the characteristics of desorption into a liquid are considerably different from the case of desorption into vacuum.

\section{APPLICATION OF TST TO DESORPTION10}

The TST rate of desorption is given by

$$
R=\frac{k T}{h} \frac{f^{*}}{f} \exp \left(-\frac{E}{k T}\right),
$$

where $k$ is the Boltzmann constant, $T$ is the absolute temperature, $f^{*}$ and $f$ are the partition functions for the activated complex and the adsorbed particle, respectively, and $E$ is the activation energy for desorption. The main difficulty with TST concerns the definition of the transition state for the desorbing species. A common choice is to assume that the transition state is located at an infinite absorbate-surface separation. For example, Garrison and Adelman ${ }^{22}$ used the TST approach to derive an expression for the one-dimen-
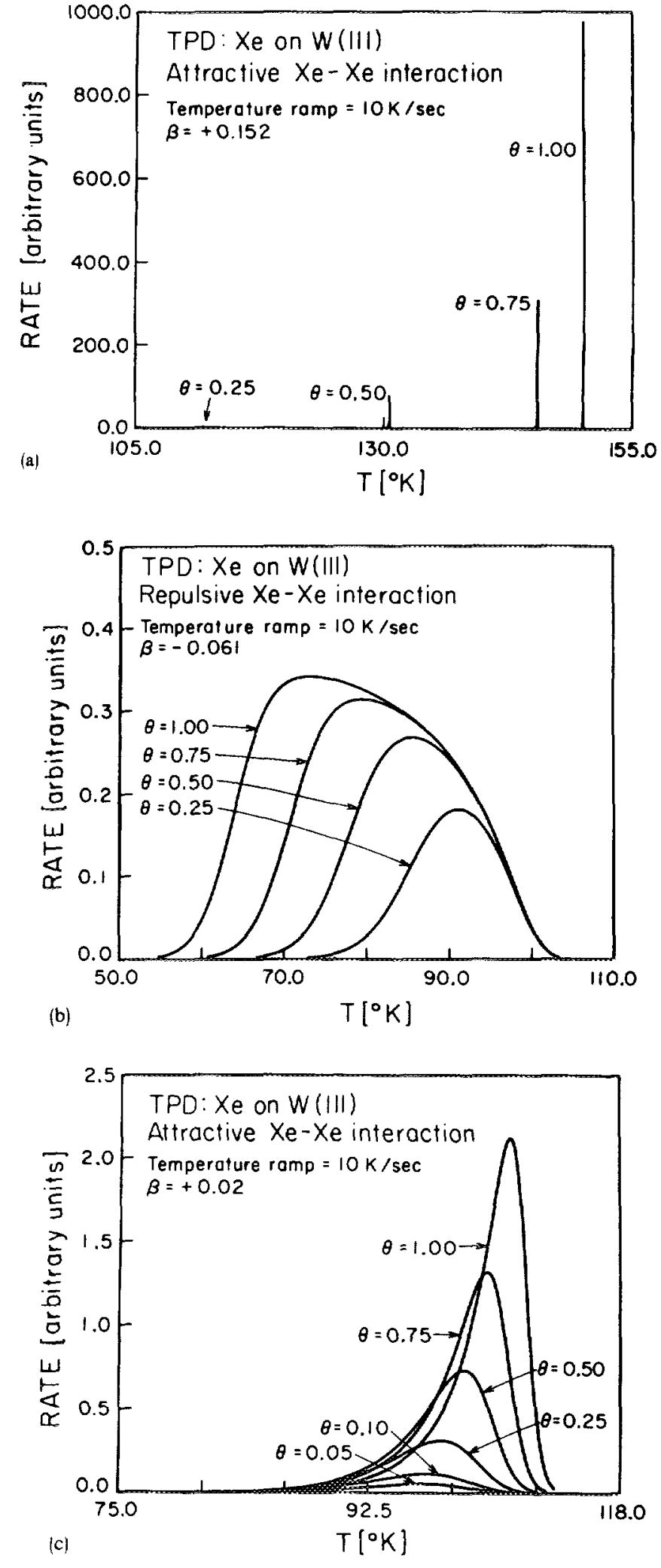

FIG. 6. Temperature programmed desorption spectra calculated for Xe on W(111). (a) Attractive Xe-Xe interaction; (b) repulsive Xe-Xe interaction; (c) attractive interaction, intermediate between (a) and (b) [after Ref. 7(c)].

sional desorption rate of an atom from a solid surface (motion of the adparticle restricted to the direction normal to the surface). In their derivation they assumed an activated complex located at an infinite distance from the substrate, leading to the partition function corresponding to a free atom 

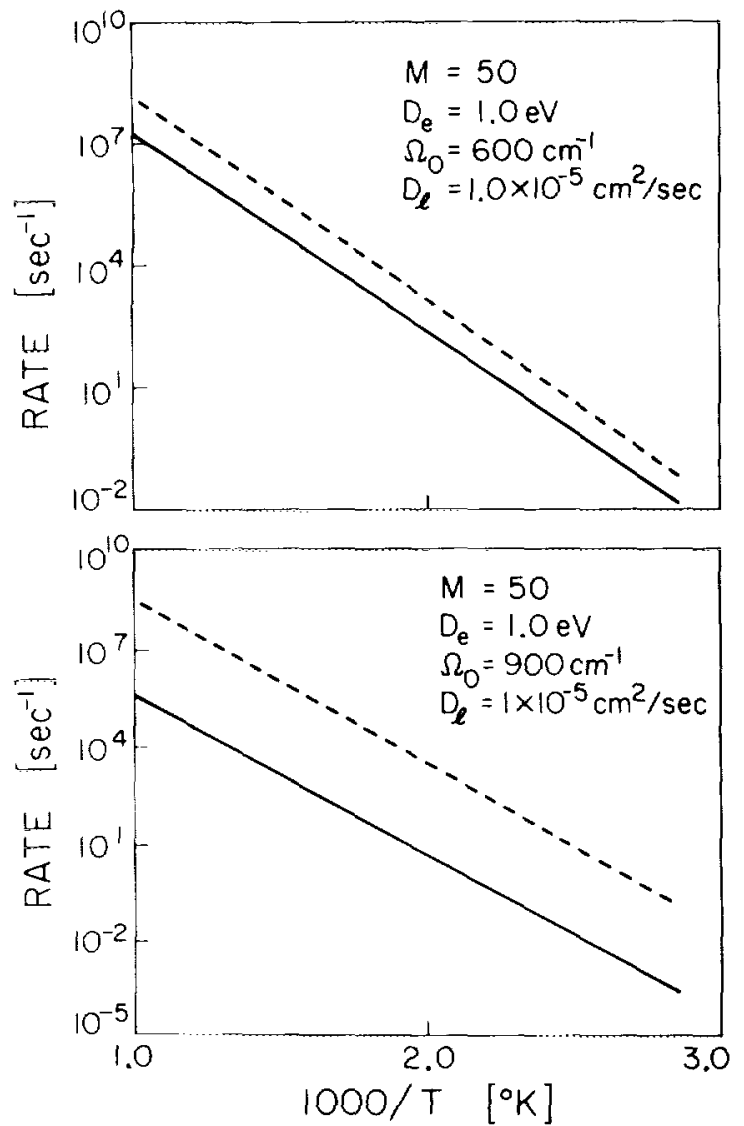

FiG. 7. Rates of desorption versus temperature for two different stretch frequencies (a) $\Omega_{0}=600 \mathrm{~cm}^{-1}$; (b) $\Omega_{0}=900 \mathrm{~cm} \quad$. The solid line corresponds to the calculated rate while the dashed line represents the rate that would be obtained by setting $r(T)=1$ (after Ref. 9).

moving in a direction normal to the surface. The rate expression obtained was

$$
R=\frac{\Omega_{0}}{2 \pi} \exp \left(-\frac{D_{e}-(1 / 2) \Omega_{0}}{k T}\right)
$$

where $\Omega_{0}$ and $D_{e}$ are the stretch frequency and binding energy associated with the absorbate-surface interaction potential. This leads to preexponential factors in the range $10^{12}$ $10^{13} \mathrm{~s}^{-1}$. On the other hand, Menzel and co-workers ${ }^{\text {(a) }}$ used the TST approach to explain the large preexponential factors obtained for the desorption rate of $\mathrm{CO}$ from metal surfaces. The rate expression derived yields a preexponential factor of the order of $10^{18} \mathrm{~s}^{-1}$ which is $10^{2}-10^{3}$ times larger than the experimentally observed values. In some cases it has been assumed ${ }^{23}$ that the ratio of the partition functions appearing in Eq. (VI.1) is approximately equal to one. As a result, considerable discrepancies (two to three orders of magnitude) with experiment have been obtained (for the case of molecular desorption).

As pointed out above, the choice of an unambiguous activated complex is of crucial importance. A criterion for the choice of the transition state is given by the variational transition state theory proposed by Horiuti. ${ }^{24}$ Here, the activated complex is defined so that the flux at the transition state is an extremum, leading to a nonlinear differential equation for the position of the critical surface. If the potential between the adparticle and the surface increases monotonically from the equilibrium position to infinity, the only solution to the
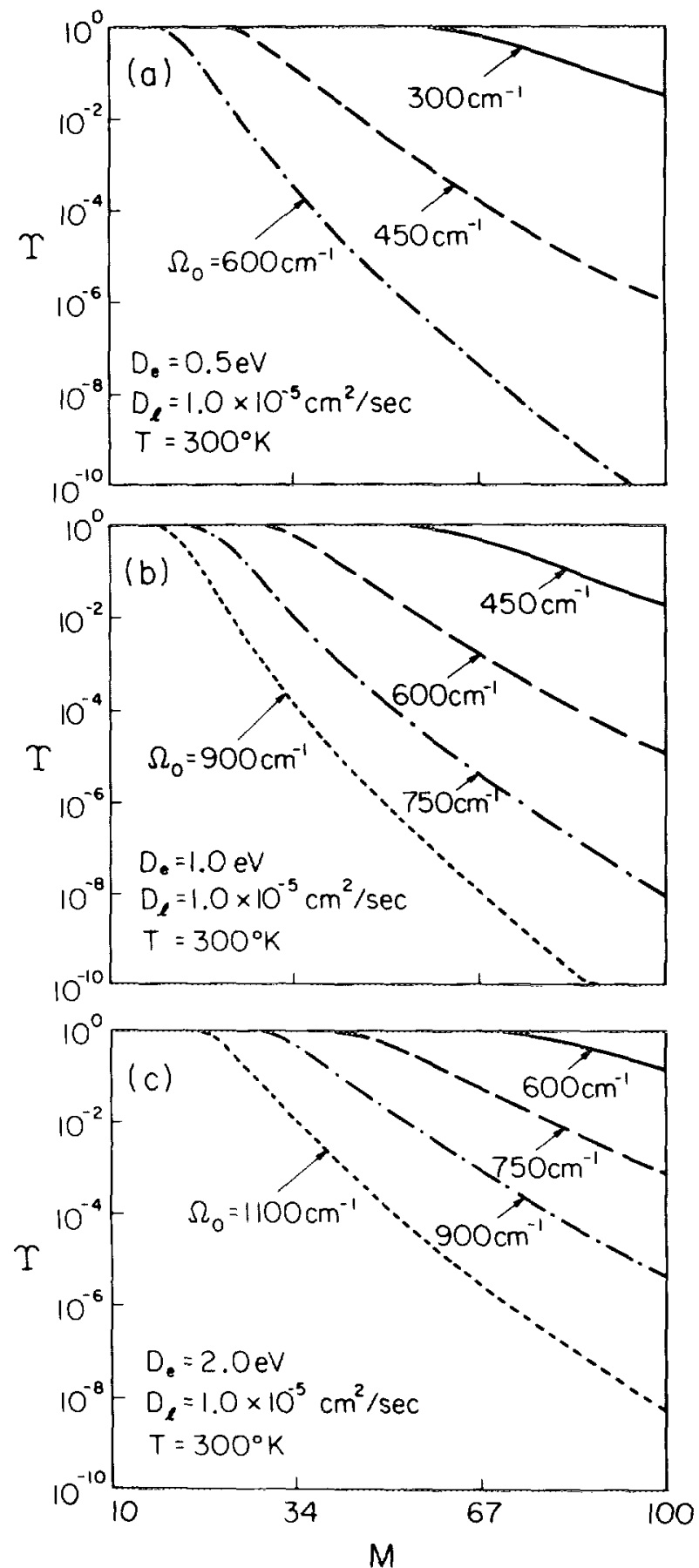

FIG. 8. Variation of $r(T)$ with the absorbate mass for different dissociation energies. (a) $D_{c}=0.5 \mathrm{eV}$; (b) $D_{c}=1.0 \mathrm{eV}$; (c) $D_{c}=2.0 \mathrm{eV}$ (after Ref. 9

variational equation corresponds to an infinite separation between the adparticle and the surface. Therefore, application of variational transition state theory leads to the activated complex geometry with the absorbate infinitely removed from the surface.

In Ref. 10 we consider an adatom of mass $m$ which is at equilibrium on the surface, at a temperature $T$. The potential near the surface is represented by

$$
V(x, y, z)=\frac{1}{2} m \Omega_{0}^{2}(x, y)\left[z-z_{0}(x, y)\right]^{2}+E(x, y),
$$

where $z_{0}$ is the equilibrium distance between the adparticle and the surface, and $E(x, y)$ describes the variation of the potential parallel to the surface (corrugation). Assuming that 
the surface is periodic in the $x$ and $y$ directions, then, $\Omega_{0}, z_{0}$, and $E$ will have the same periodicity. Then the rate of desorption is given by

$$
R=\frac{\exp \left(-D_{0} / k T\right)}{2 \pi \Sigma_{n m} \Omega_{n m}^{1} I_{n}\left(E_{x} / 2 k T\right) I_{m}\left(E_{y} / 2 k T\right)} .
$$

For the case of a flat surface $\Omega_{0}$ and $E$ are independent of $x$ and $y$, and Eq. (VI. 4) reduces to Eq. (VI.2). In the derivation of Eq. (VI.4) ${ }^{10}$ we have expanded $\Omega_{0}^{-1}$ and $E(x, y)$ in terms of Fourier series [for $E,(x, y)$ we only used the initial terms]

$$
\Omega_{0}^{-1}(x, y)=\sum_{n m} \Omega_{n m}^{-1} \cos \frac{n \pi x}{a} \cos \frac{m \pi y}{b},
$$

and

$$
E(x, y)=-\frac{1}{2} E_{x} \cos \frac{\pi x}{a}-\frac{1}{2} E_{y} \cos \frac{\pi y}{b} .
$$

The symbols $I_{n}$ and $I_{m}$ in Eq. (VI.4) stand for the modified Bessel functions of order $n$ and $m$, respectively.

The main difference between molecular and atomic desorption is due to two characteristic features of molecular absorbates. First, molecules have internal degrees of freedom that are absent in atomic systems. In addition, molecules exhibit frustrated rotational modes (surface-absorbate bending modes) strongly coupled to the surface but which become pure rotational modes in the activated state. For simplicity, consider first the case of a diatomic molecule desorbing from a surface at temperature $T$. As in the case of atomic desorption, the main contribution to the partition function arises from configurations near the equilibrium position. Hence, we shall represent the potential $V(q)$ with a form similar to that of Eq. (VI.3),

$$
\begin{aligned}
V(\mathbf{q})= & \frac{1}{2}\left\{M \Omega_{0}^{2}(x, y)\left[z-z_{0}(x, y)\right]^{2}+I_{r} \Omega_{r}^{2}(x, y) \theta^{2}\right. \\
& \left.+\mu \bar{\omega}_{i}^{2}(x, y) r^{2}\right\}+E(x, y),
\end{aligned}
$$

where $\Omega_{r}$ is the frequency associated with the bending (angle $\theta$ ) motion, and $\bar{\omega}_{i}$ is the frequency of the internal vibration at the surface. In Eq. (VI.6) we assumed that the total potential is independent of the azimuthal angle $\varphi$. This is generally a good approximation for binding sites on low Miller index surfaces but would not necessarily be valid for a molecule binding at a step or at a surface defect. The quantities depending on $x$ and $y$ will have the same periodicity as the surface; thus, they can be expanded in terms of the corresponding Fourier series. As in the atomic case, we assume that $E(x, y)$ has the first order form given in Eq. (VI.5b). The rate of desorption is then given by

$$
R=\frac{I \exp \left(-D_{0} / k T\right)}{\pi k T \omega_{i} \Sigma_{n m} \Gamma_{n m} I_{n}(E / 2 k T) I_{m}\left(E_{y} / 2 k T\right)},
$$

where $I$ is the moment of inertia. In the limit of a flat surface this expression reduces to

$$
R=\frac{\Omega_{0}}{2 \pi}\left(\frac{2 I \Omega_{r}^{2}}{k T}\right)\left(\frac{\bar{\omega}_{i}}{\omega_{i}}\right) \exp \left(-\frac{D_{0}}{k T}\right) .
$$

Except for a factor of $\pi / 4(\sim 0.8)$ this expression agrees with the corresponding rate obtained from CSDT. The difference arises from the fact that in the present treatment the integral over the angle $\theta$ was carried out from zero to infinity, whereas in the CSDT approach we integrate to a maximum bend- ing angle (which was taken to be $\pi / 2$ ). The effect of the internal vibration on the rate of desorption is given by the ratio $\bar{\omega}_{i} / \omega_{i}$. In most cases the internal vibrational frequency at the adsorbed state is only slightly different $(-10 \%-20 \%)$ from the corresponding value for a free molecule, so that this factor has a negligible effect on the desorption rate.

A comparison between the atomic and diatomic desorption rate shows that the main difference lies in the additional degrees of freedom of the diatomic molecule (e.g., frustrated rotation and internal vibration). The frustrated rotation is strongly coupled to the surface, so that the total potential which the absorbed molecule feels has a large component due to this mode. However, the corresponding mode in vacuum is a free rotation. Hence, the ratio of the partition functions in Eq. (VI.1) leads to a special (and relatively large) contribution to the prefactor.

On the other hand, the internal degrees of freedom contribute to the total potential both at the absorbed state and in the activated complex. Since the difference between these contributions to the respective partition functions is small, their effect on the preexponential factor appears as the ratio $\bar{\omega}_{i} / \omega_{i}$, which is always close to unity. Therefore, for a polyatomic molecule we can treat the contribution of the internal modes to the total interaction potential (at the surface) similarly to that of the internal vibration of a diatomic system (Eq. VI.6). Consequently, the rate of desorption of a polyatomic molecule, for the case of a flat surface, is given by

$$
R=\frac{\Omega_{0}}{2 \pi}\left(\frac{2 I \Omega_{r}^{2}}{k T}\right) \prod_{i}\left(\frac{\vec{\omega}_{i}}{\omega_{i}}\right) \exp \left(-\frac{D_{0}}{k T}\right),
$$

where the product is over all the internal degrees of freedom. The corresponding expression for the large corrugation case is

$$
\begin{aligned}
R= & \frac{\Omega_{0}}{2 \pi}\left[\frac{2 \pi I \Omega_{r}^{2}\left(E_{x} E_{y}\right)^{1 / 2}}{(k T)^{2}}\right] \prod_{i}\left[\frac{\bar{\omega}_{i}}{\omega_{i}}\right] \\
& \times \exp -\left[\frac{2 D_{0}+E_{x}+E_{y}}{2 k T}\right] .
\end{aligned}
$$

As discussed above, the rate expressions for diatomic molecules derived in this section are almost identical to those obtained using the CSDT formalism. ${ }^{25}$ In the CSDT approach we assumed that the energy stored in the frustrated rotation could be transformed (through the coupling to the surface) into transitional kinetic energy, resulting in an increase in the desorption rate (with respect to the atomic case). The agreement between those two methods confirms the validity of this assumption. Therefore, one would expect that a desorbed diatomic molecule has a rotational temperature that is about half of the surface temperature. In general, one expects that the rotational temperature of the desorbed molecule corresponds to an energy which is $\simeq(1 / 2) k T$ less than the corresponding value at the surface.

\section{CONCLUSIONS}

We find that for processes involving rates far slower than the characteristic vibrational frequencies, we can apply stochastic methods to describe the dynamics of surface and interface processes. A comparison between CSDT and TST theories for desorption suggests that it may be possible to 
apply activated complex theory to obtain similar expressions for systems that are considerably more complicated than those studied here.

As better knowledge of the potential surfaces of adsorbate species interacting with each other and with the substrate is developed, one would hope to be able to extend some of the ideas reviewed here to study such systems as associative desorption and diffusion.

Although the complications are considerably increased, we hope to extend some of the ideas of CSDT theory to the study of systems with time scales comparable to the characteristic vibrational periods (e.g., collisions, reactive scattering, epitaxial growth, etc.).

\section{ACKNOWLEDGMENTS}

This work was partially supported by the Department of Energy (Contract No. DE-AM03-76SF00767; Project Agreements Nos. DE-AT03-80ER 10608 and W-7405ENG-35).

"Chaim Weizmann Postdoctoral Fellow.

(a) C. R. Helms and R. J. Madix, Surf. Sci. 52, 677 (1975); (b) P. A. Redhead, Trans. Faraday Soc. 57, 641 (1961); (c) J. B. Pendry, Low Energy Electron Diffraction (Academic, New York, 1974); (d) L. A. Harris, J. Appl. Phys. 39, 1419 (1968); (e) for a review, see L. D. Schmidt, Catal. Rev. Sci. Eng. 9, $115(1974)$

(a) S. Glasstone. K. J. Laidler, and H. Eyring, The Theory of Rate Processes (McGraw-Hill, New York, 1941), p. 347; (b) K. J. Laidler, in Catalysis, edited by P. H. Emmett (Reinhold, New York, 1954), Vol. 1, Chap. 3; (c) A. Cordoba and J. J. Luque, Phys. Rev. B 26, 4028 (1982); 28, 7353 (1983).

(a) J. D. Doll, J Chem Phys 68, 3158 (1978); (b) E. K. Grimmelmann, J. C. Tully, and E. Helfand, ibid. 74, 5300 (1981); (c) B. J. Garrison, D. J Diestler, and S. A. Adelman, ibid. 67, 4317 (1977); (d) J. C. Tully, Surf. Sci 111, 461 (1981).

(a) G. S. De. U. Landman, and M. Rasolt, Phys. Rev. B 21, 3256 (1980); (b S. Efrima, K. F. Freed, C. Jedrzejek, and H. Metiu, Chem. Phys. Lett. 74 $43(1980)$.

(a) H. Pfnür, P. Feulner, H. A. Engelhardt, and D. Menzel, Chem. Phys Lett. 59. 481 (1978); (b) H. Ibach, W. Erley, and H. Wagner, Surf. Sci. 92 29 (1980): (c) J. L. Taylor and W. H. Weinberg, ibid. 78, 259 (1978). (a) J. E. Adams and J. D. Doll, J. Chem. Phys. 74, 1467 (1981); (b) J. L. Beeby. Surf. Sci. 114, 118 (1982).

(a) A. Redondo, Y. Zeiri, and W. A. Goddard III, Phys. Rev. Lett. 49 , 1847 (1982): (b) Y. Zeiri, A. Redondo, and W. A. Goddard III, Surf. Sci 131, 221 (1983); (c) A. Redondo, Y. Zeiri, and W. A. Goddard III, ibid. 136, $41(1984)$

(a) H. A. Kramers, Physica 7, 284(1940); (b) S. Chandrasekhar, Rev. Mod. Phys. 15, 1 (1943).

"Y. Zeiri, A. Redondo, and W. A. Goddard III, J. Electrochem. Soc. (in press).

${ }^{10}$ A. Redondo, Y. Zeiri, J. J. Low, and W. A. Goddard III, J. Chem. Phys. 79, $6410(1983)$.

"(a) S. A. Adelman and J. D. Doll, J. Chem. Phys. 61, 4242 (1974); (b) 63 4908 (1975); (c) 64, 2375 (1976); (d) Acc. Chem. Res. 10, 378 (1977); (e) S. A

Adelman, Adv. Chem. Phys. 44, 143 (1980); (f) 53, 61 (1983).

'2J. C. Tully, Ann. Rev. Phys. Chem. 31, 319 (1980)

''S. A. Adelman, J. Chem. Phys. 71, 4471 (1969).

${ }^{4} \mathrm{~J}$. N. Allison and W. A. Goddard III, Surf. Sci. 115, 553 (1982)

${ }^{5}$ P. A. Redhead, Vacuum 12, 203 (1962).

16(a) S. A. Adelman, J. Chem. Phys. 71, 447 (1979); (b) 73, 3145 (1980); (c) M. Berkowitz, C. L. Brooks III, and S. A. Adelman, ibid. 73, 3889 (1980); (d) C. L. Brooks III, M. Berkowitz, and S. A. Adelman, ibid. 73, 4353 (1980).

7) (a) L. Schmidt and R. Gomer, J. Chem. Phys. 42, 3573 (1965); (b) L. M Kahn and S. C. Ying, Solid State Commun. 16, 799 (1975); (c) G. Erlich Adv. Catal. 14, 255 (1963).

${ }^{18}$ J. N. Allison, Y. Zeiri, A. Redondo, and W. A. Goddard III, Chem. Phys. Lett. 97,387 (1983)

${ }^{14}$ See, for example: (a) M. J. Dresser, T. E. Madey, and J. T. Yates, Surf. Sci. 42, 533 (1974): (b) J T. Yates, Jr and N. E. Erickson, ibid 4A, 489 (1974), (c) G. Erlich, Adv. Catal. 14, 255 (1986); (d) T. Engel and R. Gomer, J. Chem. Phys. 52, 5572 (1970); (e) L. Schmidt and R. Gomer, ibid. 42, 3573 (1965).

2"1. A. Barker, R. O. Watts, J. K. Lee, T. P. Schaefer, and Y. T. Lee, J. Chem. Phys. 61, 3081 (1974).

"V. B. Leonas, Sov. Phys. Usp. 15, 266 (1973).

${ }^{22}$ B. J Garrison and S. A. Adelman, J. Chem. Phys. 67, 2379 (1977).

23. J) K. J. Laidler, in Catalysis, edited by P. H. Emmett (Reinhold. New York, 1945), Vol. 1, Chap. 5; (b) D. O. Hayward and B. M. W. Trapnell, Chemisorption (Butterworths, London, 1964), p. 150; (c) F. C. Tompkins, Chemisorption of Gases on Metals (Academic, London, 1978), p. 66

${ }^{24}$ (a) J. Horiuti, Bull. Chem. Soc. Jpn. 13, 210 (1938); (b) P. Pechukas, Ann. Rev. Phys. Chem. 32, 159 (1981).

${ }^{25}$ The TST approach is not capable of producing the factor $Y(T)$ appearing in the CSDT expression. Since we always have $\gamma(T) \leqslant 1$, the TST rate is an upper bound for the CSDT result. 